\title{
TRAQUEOSTOMÍA PERCUTÁNEA GUIADA POR BRONCOSCOPÍA: EXPERIENCIA EN 235 PROCEDIMIENTOS
}

\section{PERCUTANEOUS TRACHEOSTOMY GUIDED BY BRONCHOSCOPY: EXPERIENCE IN 235 PROCEDURES TRAQUEOSTOMIA PERCUTÂNEA GUIADA POR BRONCOSCOPIA: EXPERIÊNCIA EM 235 PROCEDIMENTOS}

\author{
Indalecio Carboni Bisso ${ }^{1,3}$, Iván Huespe ${ }^{1}$, Sofía Schverdfinger ${ }^{1}$, Carlos Videla ${ }^{1}$, Juan Montagne ${ }^{2}$, Agustín Dietrich², David \\ Smith², Eduardo San Román ${ }^{1}$, Marcos Las Heras'.
}

\begin{abstract}
La traqueostomía es un procedimiento quirúrgico realizado con el objetivo de crear una abertura dentro de la tráquea, a través de una incisión ejecutada en el cuello, para insertar una cánula que facilite el paso de aire a los pulmones. Muchas veces, es necesario realizar una traqueostomía cuando, por problemas de salud, el paciente tiene que usar una máquina (respirador) por un tiempo prolongado para ayudarle a respirar.

Existen diferentes técnicas para realizar el procedimiento. Entre ellas, la técnica de traqueostomía percutánea ofrece múltiples ventajas ya que se trata de un procedimiento mínimamente invasivo, que requiere un tiempo quirúrgico corto, y sobre todo, puede realizarse a la cabecera del paciente sin requerir un traslado a quirófano. En este estudio evaluamos la tasa de complicaciones asociadas a la realización de traqueostomía percutánea describiendo la técnica utilizada en nuestro hospital que incluye el uso de un broncoscopio para guiar al cirujano. Nuestro objetivo fue conocer la efectividad $y$ seguridad de este procedimiento en terapia intensiva, con buenos resultados.
\end{abstract}

\section{Conceptos clave:}

¿Qué se sabe sobre el tema? La traqueostomía es un procedimiento quirúrgico frecuente en las unidades de cuidados intensivos. La técnica percutánea ofrece ventajas tales como evitar traslados potencialmente peligrosos fuera de la UCI y una menor tasa de complicaciones infecciosas. En los últimos años, debido a la mayor disponibilidad de instrumental, el uso de la videobroncoscopia como guía durante el procedimiento posibilita visualizar directamente la vía aérea, al tiempo que permite intervenir rápidamente en caso de hemorragia y reduce el riesgo en pacientes con anatomía aberrante o de difícil palpación.

¿Qué aporta este trabajo? Registra la eficacia y complicaciones asociadas a la a realización de traqueostomía percutánea guiada por broncoscopia en un hospital universitario de alta complejidad. Los resultados de este estudio evidencian una menor tasa de complicaciones en comparación con series internacionales de TP con guía endoscópica reportadas previamente. Asimismo, en nuestro conocimiento, incluye el mayor número de casos reportado a nivel nacional, lo que podría aportar valor en el área para conformar estadísticas en nuestro medio.

1-Terapia Intensiva de Adultos, Hospital Italiano de Buenos Aires - Cirugía de tórax, Hospital Italiano de Buenos Aires

3- E-mail de contacto: indalecio.carbonibisso@hospitalitaliano.org.ar

Recibido: 2020-05-05 Aceptado: 2020-06-05

DOI: http://dx.doi.org/10.31053/1853.0605.v77.n3.28474

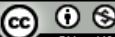

(C) Universidad Nacional de Córdoba

\section{Resumen:}

Objetivo: Medición de la eficacia y relevamiento de las complicaciones asociadas a la a realización de traqueostomía percutánea (TP) guiada por videobroncoscopia en un hospital universitario de alta complejidad.

Materiales y métodos: Estudio observacional retrospectivo realizado entre mayo de 2017 y agosto de 2019. El criterio para la indicación de TP fue desvinculación prolongada de la ventilación mecánica (DPVM) en todos los casos. Incluyó pacientes mayores de 18 años en que se realizó TP electiva guiada por videobroncoscopia. Se registraron variables demográficas, APACHE II y días de ventilación mecánica previos a la TP. La eficacia del procedimiento fue evaluada en base a la tasa de éxito en la ejecución, la necesidad de conversión a técnica abierta. Asimismo, se registraron las complicaciones observadas.

Resultados: Se evaluaron 235 procedimientos (149 hombres y 86 mujeres) en pacientes con una mediana de edad de 65 años (rango intercuartílico 50 - 74), un score APACHE II de 18 (rango intercuartílico 12 - 24). La TP pudo ser ejecutada en forma rápida y satisfactoria en todos los pacientes sin requerimiento de conversión a técnica abierta. Se presentaron complicaciones tempranas en el 3,8\% (9) de los casos. El sangrado menor fue la complicación más frecuentemente observada en 5 casos $(2,1 \%)$, hipotensión en 3 casos $(1,3 \%)$ e hipoxemia transitoria en un caso (0,4\%). Así mismo el 2,1\% (5) de los casos registró complicaciones tardías. Conclusión: La realización de TP mediante la técnica de dilatador único guiada por videobroncoscopia se describe como un procedimiento efectivo y seguro, que puede realizarse en unidades de cuidados intensivos con baja tasa de complicaciones.

Palabras clave: traqueostomía; broncoscopia; cuidados intensivos.

Abstract:

Objective: Measurement of the efficacy and complications associated with performing percutaneous tracheostomy (PT) guided by video bronchoscopy.

Materials and methods: Retrospective observational study conducted between May 2017 and August 2019. Adult patients who underwent elective PT guided by video bronchoscopy were included. The criteria for the indication of PT was prolonged weaning from mechanical ventilation in all cases. Demographic variables, APACHE II score and days of mechanical ventilation prior to PT were recorded. The efficacy of the procedure was evaluated based on the success rate in the execution, the need for conversion to open technique. Also complications observed were recorded.

Results: 235 procedures (149 men and 86 women) were evaluated in patients with an average age of 65 years (interquartile range 50 - 74) APACHE II score 18 (interquartile range 12 - 24). The PT was performed quickly and satisfactorily in all patients without conversion to open technique in any case. Complications occurred in 3.8\% (9) of the cases. Minor bleeding was the most frequently observed complication in 5 cases $(2.1 \%)$, hypotension in 3 cases $(1.3 \%)$, and transient hypoxemia in one patient $(0.4 \%)$. Also, $2,1 \%(5)$ of the cases presented late complications.

Conclusion: Performing PT guided by video bronchoscopy is described as an effective and safe procedure that can be done in intensive care units with a low rate of complications.

Keywords: tracheostomy; bronchoscopy; critical care.

\section{Resumo}

Objetivo: Medir a eficácia e as complicações associadas à realização de traqueostomia percutânea (TP) guiada por videobroncoscopia.

Materiais e métodos: Estudo observacional retrospectivo realizado entre maio de 2017 e agosto de 2019. Foram incluídos pacientes adultos submetidos a TP eletivo guiado por vídeo broncoscopia. Os critérios para indicação de TP foram desmame prolongado da ventilação mecânica em todos os casos. Foram registradas variáveis demográficas, escore APACHE II e dias de ventilação mecânica antes do TP. A eficácia do procedimento foi avaliada com base na taxa de sucesso na execução, na necessidade de conversão para a técnica aberta. Também foram observadas complicações observadas.

Resultados: foram avaliados 235 procedimentos (149 homens e 86 mulheres) em pacientes com idade mediana de 65 anos (intervalo intercuartil 50 - 74), un score APACHE II de 18 (intervalo intercuartil 12 - 24). O TP foi realizada de forma rápida e satisfatória em todos os pacientes sem conversão para técnica aberta. Complicações ocorreram em 3,8\% (9) dos casos. Sangramento menor foi a complicação mais frequentemente observada em 5 casos (2,1\%), hipotensão em 3 casos $(1,3 \%)$ e hipoxemia transitória em um paciente $(0,4 \%)$. Além disso, $2,1 \%(5)$ dos casos apresentaram complicações tardias.

Conclusão: A realização de TP guiada por vídeo broncoscopia é descrita como um procedimento eficaz e seguro que pode ser realizado em unidades de terapia intensiva com baixo índice de complicações. 


\section{Introducción}

La técnica de traqueostomía percutánea (TP) fue descrita originalmente por Shelden ${ }^{1}$ en 1955, sin embargo, estuvo asociada a una elevada tasa de complicaciones lo que limitó su popularización. Hacia 1985, Ciaglia ${ }^{2}$ desarrolló un método con técnica de Seldinger que facilitó la introducción de dispositivos de diferente calibre para dilatar progresivamente la tráquea hasta la canulación. Finalmente, en 1998, se introdujo una modificación al procedimiento reemplazando el sistema de dilatadores múltiples por un dilatador único con un perfil de seguridad superior ${ }^{3}, 4$. Por los beneficios que ofrece sobre la técnica quirúrgica tradicional, esta técnica comenzó a utilizarse de forma masiva.

Actualmente, la traqueostomía es un procedimiento quirúrgico frecuente en las unidades de cuidados intensivos (UCI). Se indica como estrategia de desvinculación en pacientes que requieren ventilación mecánica prolongada para prevenir las potenciales complicaciones de la intubación orotraqueal, tales como neumonía asociada a la ventilación mecánica, sinusitis y estenosis traqueal. Asimismo, confiere un aumento en la comodidad del paciente, mejora la higiene bronquial y permite disminuir el requerimiento de sedación profunda ${ }^{5,6}$. No obstante, su impacto en la morbi-mortalidad aún es motivo de debate ${ }^{7}$.

En el contexto del paciente crítico, la TP frente a la técnica quirúrgica ofrece ventajas tales como evitar traslados potencialmente peligrosos fuera de la $\mathrm{UCl}$, una menor tasa de complicaciones infecciosas y disminución en los costos para el sistema de salud ${ }^{8}$. Cabe señalar que las guías de práctica clínica basadas en la evidencia actual no recomiendan una modalidad de traqueostomía sobre otra, sin embargo, la planificación cuidadosa del procedimiento, así como el desarrollo y perfeccionamiento de estrategias más seguras, son esenciales para minimizar complicaciones. En los últimos años, debido a la mayor disponibilidad de instrumental, el uso de la videobroncoscopia en $\mathrm{UCl}$ se ha generalizado, posibilitando la visualización directa de la vía aérea, al tiempo que permite intervenir rápidamente en caso de hemorragia y reduce el riesgo en pacientes con anatomía aberrante o de difícil palpación ${ }^{9}$.

El objetivo de este estudio es medir la eficacia y registrar las complicaciones asociadas a la a realización de TP guiada por videobroncoscopia en un hospital universitario de alta complejidad.

\section{Métodos}

Estudio observacional retrospectivo realizado en el periodo de mayo de 2017 a agosto de 2019. Se evaluaron pacientes mayores de 18 años internados en las $\mathrm{UCl}$ de un hospital universitario de alta complejidad (Terapia Intensiva de Adultos, Terapia Intermedia de Adultos y Unidad de Cuidados Intensivos Coronarios). Fueron incluidos los datos de los pacientes en los que se realizó TP electiva guiada por videobroncoscopia. El criterio para la indicación de TP fue desvinculación prolongada de la ventilación mecánica (DPVM) que es el proceso que requiere más de tres pruebas de ventilación espontánea o un periodo de weaning de más de siete días desde la primera prueba de ventilación espontánea, evaluado en cada caso por el equipo médico de la $\mathrm{UCl}{ }^{10}$. Los pacientes con severas deformidades anatómicas de cuello fueron excluidos ya que en este grupo se realizó traqueostomía con técnica quirúrgica fuera de la UCl.

Se registraron variables demográficas tales como edad, sexo, score APACHE I| y mortalidad hospitalaria de la serie; motivo de internación; días de internación en UCl; días en ventilación mecánica hasta el procedimiento; complicaciones observadas; y tiempo de duración del procedimiento. Este último fue registrado como tiempo anestésico y tiempo quirúrgico. El tiempo anestésico se definió como aquel período de tiempo durante el cual se requirió un aumento o cambio en la sedoanalgesia para alcanzar un objetivo de Richmond Analgesia Sedation Scale (RASS) -5. Una vez finalizado el procedimiento, se restableció de sedoanalgesia esquema previo. Por otra parte, el tiempo quirúrgico se contabilizó desde la colocación del campo quirúrgico hasta la conexión de la cánula de traqueostomía al ventilador mecánico.
La eficacia en la ejecución de la TP fue evaluada en base a la tasa conversión a técnica abierta o la necesidad de suspender el procedimiento.

Debido a que no existen guías que evalúen la seguridad de la técnica de TP, se utilizó como referencia la guía de la Sociedad Británica de Tórax de broncoscopia (del inglés, British Thoracic Society) ${ }^{11}$ para definir las complicaciones tempranas (durante el acto quirúrgico y hasta 5 días luego de realizado el mismo $)^{12}$ :

- Desaturación: disminución mayor al $4 \%$ en la saturación de oxígeno (SpO2) por pulsioximetría o $\mathrm{SpO} 2$ menor a $90 \%$, por más de un minuto.

- Arritmias: aumento o disminución del $40 \%$ en la frecuencia cardíaca respecto a la frecuencia cardíaca de inicio, frecuencia cardíaca mayor a 110 latidos por minuto, frecuencia cardíaca menor a 50 latidos por minuto, y/o cambios en el ritmo cardíaco evidenciados en el monitoreo electrocardiográfico continuo.

- Hipertensión: aumento del $30 \%$ en la tensión arterial respecto a la tensión arterial de inicio.

- Hipotensión: disminución del $30 \%$ en la tensión arterial respecto de la tensión arterial de inicio o tensión arterial media menor de $65 \mathrm{mmHg}$.

- $\quad$ Sangrado

Sin sangrado: trazos de sangre que no requieren aspiración continua.

Sangrado menor: sangrado que requiere aspiración continua de la vía aérea. El sangrado cede espontáneamente.

Sangrado moderado: sangrado que requiere maniobras hemostáticas con el endoscopio tales como infusión de adrenalina, ácido tranexámico o solución salina fría.

Sangrado mayor: sangrado que requiere resucitación con cristaloides, transfusiones o suspensión del procedimiento.

Asimismo, otras complicaciones como el retiro no programado de tubo orotraqueal, lesión de la vía aérea, punción esofágica, neumotórax y neumomediastino fueron consideradas.

Se realizó un seguimiento de los pacientes hasta el alta hospitalaria con el objetivo de registrar las complicaciones tardías, definidas como aquellas complicaciones que aparecieron luego de los 5 días de realizada la TP ${ }^{12}$.

Durante la confección de la TP se configuró, en todos los casos, el ventilador mecánico en modo volumen control para obtener una ventilación a $8 \mathrm{ml} / \mathrm{kg}$ de peso teórico, con una $\mathrm{FiO}_{2}$ al 100\%, bajo sedación profunda con ketamina (bolo de $2 \mathrm{mg} / \mathrm{kg}$ ) y propofol en infusión continua para mantenimiento (dosis de $1,8 \mathrm{mg} / \mathrm{kg} / \mathrm{h}$ ), asociado a bloqueo neuromuscular con rocuronio (bolo de $1 \mathrm{mg} / \mathrm{kg}$ ). El procedimiento broncoscópico estuvo a cargo de dos tipos de operadores: 1) médicos especialistas en broncoscopia; 2) médicos especialistas en cuidados intensivos en un programa de formación en broncoscopia. El procedimiento percutáneo fue realizado por un cirujano de tórax. Se introdujeron modificaciones a la técnica de Ciaglia Blue Rhino descrita clásicamente. Esta técnica modificada consta de cervicotomía transversa de 2 centímetros por encima de la horquilla esternal, con posterior disección de tejido celular subcutáneo con electrobisturí con una incisión vertical y apertura del músculo platisma con maniobra digital para evitar lesionar las fibras musculares. Luego se palpan los anillos traqueales, eligiendo el sitio de punción entre segundo y tercer cartílago. Posteriormente se procede a la punción traqueal bajo visión directa utilizando aguja calibre 14 Gauge, verificando que la punción se ubique en la línea media con indemnidad de la pars membranosa traqueal mediante videobroncoscopia. A continuación se progresa el alambre guía y se dilata la cara anterior de la tráquea con dilatador único según técnica de Seldinger. Una vez realizada la dilatación, se coloca la cánula de traqueotomía. Finalmente, se realiza control broncoscópico a través de ésta cánula para corroborar el correcto posicionamiento y aspirar secreciones o restos hemáticos.

En todos los procedimientos se utilizó un videobroncoscopio descartable (Ambu ${ }^{\circledR}$ aScope ${ }^{\mathrm{TM}} 3$ Regular 5.0/2.2), un set de TP con kit de dilatador único (TRACOE ${ }^{\circledR}$ experc ${ }^{\mathrm{TM}}$ ) y un generador 
electroquirúrgico (Covidien ${ }^{\circledR}$ Force $\mathrm{FX}^{\mathrm{TM}}$ ) con electrobisturí (Covidien ${ }^{\circledR}$ Rocker switch pencil ${ }^{\mathrm{TM}}$ ).

\section{Análisis estadístico}

Las variables continuas se expresaron como medianas e intervalos intercuartílicos. Las variables categóricas se resumieron como recuentos y porcentajes. Debido a que la cohorte de pacientes en nuestro estudio no se derivó de la selección aleatoria, todas las estadísticas se consideran solo descriptivas. Todos los análisis se realizaron con el uso del software $R$, versión 3.6.2 (R Foundation for Statistical Computing).

\section{Resultados}

Entre mayo de 2017 a agosto de 2019, 23182 pacientes cursaron internación en las UCl, de los cuales 5022 requirieron ventilación mecánica. Entre ellos 285 presentaron DPVM. Se realizaron 235 TP ya que 31 pacientes presentaban adecuación de los cuidados terapéuticos y a 19 pacientes se les realizó traqueostomía quirúrgica por deformaciones anatómicas severas a nivel del cuello.

Fueron incluídos los resultados de 235 pacientes (149 hombres y 86 mujeres) con una mediana de edad de 65 años (rango intercuartílico 50 - 74), un score APACHE II de 18 (rango intercuartílico 12 - 24), y una mortalidad hospitalaria de la serie del 28,9 \% (68). Las características de la población están detalladas en la Tabla 1.

Tabla $\mathbf{N}^{\circ}$ 1: Características poblacionales.

\begin{tabular}{l|c}
\hline \multicolumn{1}{c|}{ Número de pacientes, $\mathbf{n}$} & $\mathbf{2 3 5}$ \\
\hline Sexo masculino, n (\%) & $149(63,4 \%)$ \\
Edad, mediana (rango intercuartílico) & $65(50-74)$ \\
Score APACHE II, mediana (rango intercuartílico) & $18(12-24)$ \\
Días de UCI, mediana (rango intercuartílico) & $36(25-54)$ \\
Días de internación, mediana (rango intercuartílico) & $40(31-48)$ \\
Días de ventilación mecánica previos a la TP, & $9(6-12)$ \\
mediana (rango intercuartílico) & \\
\hline Motivo de ingreso*, n (\%) & $74(31,4 \%)$ \\
\hline Respiratorio & $54(22,9 \%)$ \\
Neurológico & $51(21,1 \%)$ \\
Cardiovascular & $38(16,1 \%)$ \\
Abdominal & $18(7,6 \%)$ \\
Politraumatismo & \\
\hline * Se listan los motivos de ingreso &
\end{tabular}

quirúrgicas. Politraumatismo se lista separadamente por afectar múltiples sistemas en forma concomitante. UCI: Unidad de cuidados intensivos.

La mediana de tiempo de ventilación mecánica previo al procedimiento fue de 9 días (rango intercuartílico 6 - 12).

La TP pudo ser ejecutada en forma rápida y satisfactoria en todos los pacientes sin requerimiento de conversión a técnica abierta, con una mediana de tiempo anestésico de 21,5 minutos (rango intercuartílico 16 - 25) y de tiempo quirúrgico de 10,8 (rango intercuartílico 8 - 13,7). Asimismo, no se requirió suspensión del procedimiento en ningún caso.

Se presentaron complicaciones tempranas en el 3,8\% (9) de los casos. El sangrado menor fue la complicación más frecuentemente observada en el $2,1 \%$ (5) de los casos. Otras complicaciones fueron hipotensión [1,3\% (3)] asociada a la sedación e hipoxemia transitoria en un caso $[0,4 \%(1)]$, que cedió al retirar el fibroscopio del tubo orotraqueal. No se registraron eventos graves tales como neumotórax, neumomediastino, pérdida o lesión vía aérea.

Se registraron complicaciones tardías en el 2,1\% (5) de los casos. 3 casos $(1,3 \%)$ presentaron estenosis traqueal de diferente severidad, uno de ellos necesitó desobstrucción endoscópica, y los restantes no requirieron tratamiento. Otra complicación observada fue la infección del estoma en 2 casos $(0,9 \%)$.

El procedimiento broncoscópico fue realizado por médicos especialistas en cuidados intensivos en un programa de formación en broncoscopia en el $78,7 \%$ (185) de los casos. El 21,3\% (50) restante fue realizado por médicos especialistas en broncoscopia, tanto neumonólogos como intensivistas.

\section{Conclusión}

En nuestra experiencia, la confección de TP realizada bajo guía endoscópica en $\mathrm{UCl}$ es un procedimiento eficaz y seguro. La ausencia de complicaciones graves tales como neumotórax, neumomediastino, retiro no programado del tubo orotraqueal, lesión vía aérea y punción esofágica, es atribuible al uso de la videobroncoscopia y a la seguridad que confiere la técnica percutánea.

Los resultados de este estudio evidencian una menor tasa de complicaciones en comparación con series internacionales de TP con guía endoscópica reportadas previamente ${ }^{9,13,14}$. Se encuentran resultados similares a la mayor serie de pacientes reportada a nivel latinoamericano en un estudio prospectivo de 10 años ${ }^{15}$. Esta diferencia podría ser explicada por la falta de una estandarización universal en las definiciones de las complicaciones, así como la especialización alcanzada en el procedimiento en los distintos centros.

Una limitación de nuestro estudio se centra en la falta de seguimiento luego del alta hospitalaria, y consecuentemente en el déficit de registro de las complicaciones tardías de aparición posterior. Sin embargo, éstas complicaciones no siempre son atribuibles al procedimiento de confección de traqueostomía sino también a factores precipitantes como desnutrición, falta de control de la presión del balón de neumotaponamiento, lesiones durante la intubación orotraqueal, etcétera.

Otra limitante del estudio es que se presenta la experiencia de un único centro en forma observacional y retrospectiva, y no se incluye un grupo control, sin embargo el número de pacientes críticos considerados es el mayor reportado a nivel nacional hasta el momento lo cual aporta conocimiento de valor para el área y conforma estadísticas locales. Sería importante desarrollar un estudio a futuro con objetivos comparables, en distintos hospitales con operadores y equipamiento uniforme.

Finalmente, teniendo en cuenta que la técnica es operador dependiente, creemos que la capacitación y experiencia de los operadores juega un papel importante y determina que un procedimiento como el descripto tenga la eficacia y seguridad detallada.

Limitaciones de responsabilidad

Todos los autores han contribuido a la preparación del trabajo, reúnen las condiciones de autoría y han aprobado la versión final del mismo.

Fuentes de apoyo

No existen conflictos de intereses con otros autores, instituciones, laboratorios, profesionales $u$ otros. Asimismo, tampoco contamos con fuentes de apoyo externas.

\section{Originalidad del trabajo}

Declaramos que el trabajo es original y no ha sido previamente publicado ni está en proceso de revisión por ninguna otra revista.

\section{Cesión de derechos}

Declaramos que se ceden los derechos de autor a la Universidad Nacional de Córdoba para publicar en la Revista de la Facultad de Ciencias Médicas de Córdoba y para la traducción al inglés del manuscrito por parte de la RFCM.

Participación de los autores

Todos los autores han contribuido a la preparación del trabajo, reúnen las condiciones de autoría y han aprobado la versión final del mismo. 


\section{Bibliografía}

1. Shelden Ch, Pudenz Rh, Freshwater Db, Crue BI. A new method for tracheotomy. J Neurosurg. 1955 Jul;12(4):428-31. doi: 10.3171/jns. 1955.12.4.0428.

2.Ciaglia $P$, Firsching $R$, Syniec $C$. Elective percutaneous dilatational tracheostomy. A new simple bedside procedure; preliminary report. Chest. 1985 Jun;87(6):715-9. doi: 10.1378/chest.87.6.715.

3. Johnson JL, Cheatham ML, Sagraves SG, Block EF, Nelson LD. Percutaneous dilational tracheostomy: a comparison of singleversus multiple-dilator techniques. Crit Care Med. 2001 Jun;29(6):1251-4. doi: 10.1097/00003246-200106000-00036.

4. Byhahn C, Westphal K, Meininger D, Gürke B, Kessler P, Lischke $V$. Single-dilator percutaneous tracheostomy: a comparison of PercuTwist and Ciaglia Blue Rhino techniques. Intensive Care Med. 2002 Sep;28(9):1262-6. doi: 10.1007/s00134-002-1405-4.

5.Stauffer JL, Olson DE, Petty TL. Complications and consequences of endotracheal intubation and tracheotomy. A prospective study of 150 critically ill adult patients. Am J Med. 1981 Jan;70(1):65-76. doi: 10.1016/0002-9343(81)90413-7.

6.Szakmany $T$, Russell P, Wilkes AR, Hall JE. Effect of early tracheostomy on resource utilization and clinical outcomes in critically ill patients: meta-analysis of randomized controlled trials. $\mathrm{Br} J$ Anaesth. 2015 Mar;114(3):396-405. doi: 10.1093/bja/aeu440.

7. Siempos II, Ntaidou TK, Filippidis FT, Choi AMK. Effect of early versus late or no tracheostomy on mortality and pneumonia of critically ill patients receiving mechanical ventilation: a systematic review and meta-analysis. Lancet Respir Med. 2015 Feb;3(2):150-158. doi: 10.1016/S2213-2600(15)00007-7.

8. Raimondi N, Vial MR, Calleja J, Quintero A, Cortés Alban A, Celis E, Pacheco C, Ugarte S, Añón JM, Hernández G, Vidal E, Chiappero G, Ríos F, Castilleja F, Matos A, Rodriguez E, Antoniazzi $P$, Teles JM, Dueñas C, Sinclair J, Martínez L, Von der Osten I, Vergara J, Jiménez E, Arroyo M, Rodriguez C, Torres J, Fernandez-Bussy S, Nates JL. Evidence-based guides in tracheostomy use in critical patients. Med Intensiva. 2017 Mar;41(2):94-115. English, Spanish. doi: 10.1016/j.medin.2016.12.001.

9. Shen $G$, Yin $H$, Cao $Y$, Zhang $M, W u$ J, Jiang $X, Y u T$, Lu W. Percutaneous dilatational tracheostomy versus fibre optic bronchoscopy-guided percutaneous dilatational tracheostomy in critically ill patients: a randomised controlled trial. Ir J Med Sci. 2019 May;188(2):675-681. doi: 10.1007/s11845-018-1881-3.

10. Boles JM, Bion J, Connors A, Herridge M, Marsh B, Melot $C$, Pearl R, Silverman $H$, Stanchina M, Vieillard-Baron A, Welte T. Weaning from mechanical ventilation. Eur Respir J. 2007 May;29(5):1033-56. doi: 10.1183/09031936.00010206.

11. Du Rand IA, Blaikley J, Booton R, Chaudhuri N, Gupta V, Khalid S, Mandal S, Martin J, Mills J, Navani N, Rahman NM, Wrightson JM, Munavvar M; British Thoracic Society Bronchoscopy Guideline Group. British Thoracic Society guideline for diagnostic flexible bronchoscopy in adults: accredited by NICE. Thorax. 2013 Aug;68 Suppl 1:i1-i44. doi: 10.1136/thoraxjnl-2013-203618.

12. Lerner $A D$, Yarmus L. Percutaneous Dilational Tracheostomy. Clin Chest Med. 2018 Mar;39(1):211-222. doi: 10.1016/j.ccm.2017.11.009.

13. Saritas A, Saritas PU, Kurnaz MM, Beyaz SG, Ergonenc T. The role of fiberoptic bronchoscopy monitoring during percutaneous dilatational tracheostomy and its routine use into tracheotomy practice. J Pak Med Assoc. 2016 Jan;66(1):83-9.

14. Romero P C, Cornejo R R, Ruiz C M, Gálvez A R, Llanos $\checkmark$ O, Tobar A E, Larrondo G J, Castro O J. Traqueostomía percutánea con asistencia fibrobroncoscópica: Evaluación prospectiva de 100 casos consecutivos y revisión de la literatura [Fiberoptic bronchoscopy assisted percutaneous tracheostomy: report of 100 patients]. Rev Med Chil. 2008 Sep;136(9):1113-20.

15. Romero CM, Cornejo R, Tobar E, Gálvez R, Luengo C, Estuardo N, Neira R, Navarro JL, Abarca O, Ruiz M, Berasaín
MA, Neira W, Arellano D, Llanos O. Fiber optic bronchoscopyassisted percutaneous tracheostomy: a decade of experience at a university hospital. Rev Bras Ter Intensiva. 2015 AprJun;27(2):119-24. doi: 10.5935/0103-507X.20150022. 